Archives de sciences sociales des religions

109 | janvier-mars 2000

Formes religieuses caractéristiques de

l'ultramodernité : France, Pays-Bas, États-Unis, Japon, analyses globales

\title{
Les nouvelles voies spirituelles au Japon : état des lieux et mutations de la religiosité
}

Jean-Pierre Berthon et Naoki Kashio

\section{OpenEdition \\ Journals}

Édition électronique

URL : http://journals.openedition.org/assr/20176

DOI : $10.4000 /$ assr.20176

ISSN : $1777-5825$

Éditeur

Éditions de l'EHESS

Édition imprimée

Date de publication : 1 mars 2000

Pagination : 67-85

ISBN : 2-222-96690-6

ISSN : 0335-5985

Référence électronique

Jean-Pierre Berthon et Naoki Kashio, «Les nouvelles voies spirituelles au Japon : état des lieux et mutations de la religiosité », Archives de sciences sociales des religions [En ligne], 109 | janvier-mars 2000, mis en ligne le 19 août 2009, consulté le 30 avril 2019. URL : http://journals.openedition.org/ assr/20176; DOI : 10.4000/assr.20176

Ce document a été généré automatiquement le 30 avril 2019

(C) Archives de sciences sociales des religions 


\title{
Les nouvelles voies spirituelles au Japon : état des lieux et mutations de la religiosité
}

\author{
Jean-Pierre Berthon et Naoki Kashio
}

\section{Les jalons religieux de la modernité}

1 Le processus de "sécularisation » à l'œuvre dans la société japonaise ne peut s'analyser sans tenir compte de la spécificité de l'univers religieux d'un pays où les systèmes idéoreligieux sont pluriels et où, depuis l'introduction des premiers courants religieux d'origine continentale au milieu du $\mathrm{VI}^{\mathrm{e}}$ siècle, le syncrétisme - savant ou populaire - a façonné la culture religieuse de l'archipel, même si, au fil des siècles, une différenciation fonctionnelle entre bouddhisme et shintô s'est mise en place: au premier, les cultes funéraires et la gestion des ancêtres; au second, la naissance, la reproduction et la fertilité.

2 Dans la deuxième partie du XIX siècle, le Japon se trouve confronté à de profonds bouleversements socio-politiques qui aboutissent à ce qu'il est convenu d'appeler «la restauration de Meiji » (Meiji ishin). La transformation socioculturelle de la religion (sa neutralisation et sa relativisation) peut, globalement, à partir de l'établissement de l'ère Meiji (1868), se diviser en trois périodes.

3 La première période débute en 1868 (première année de l'ère Meiji) et s'étend jusqu'à la fin de la Seconde Guerre mondiale. Au cours de cette époque, sous couvert d'attenter à l'ordre social et accusées de crime de lèse-majesté, les nouvelles religions japonaises sont persécutées par un gouvernement qui érige le shintô en religion d'État. La moindre transgression d'un système religieux à l'intérieur duquel, seuls treize mouvements obtiennent, peu à peu, sous le terme de "shintô des sectes", une reconnaissance officielle, débouche sur des sanctions immédiates d'une extrême sévérité ${ }^{1}$, tout spécialement à partir des années trente où la domination du shintô d'État s'accentue, parallèlement à la prégnance du culte impérial. En dépit de ces conditions défavorables, 
les religions populaires existantes se maintiennent et de nombreuses autres voient même le jour.

4 La deuxième période couvre les années cinquante et les années soixante. Après la guerre, les persécutions gouvernementales sur les religions (en particulier, sur les nouvelles religions) cessent. Sous la pression américaine, la séparation stricte du religieux et du politique est imposée et le shintô d'État démantelé. C'est l'occasion de noter que cette coupure entre l'État et la religion n'a été ni décidée, ni réalisée par le peuple japonais. Cependant, ce dernier a pu ainsi obtenir la liberté religieuse par la Loi sur les religions de 1951. Pour la population japonaise, cette séparation signifie avant tout l'interdiction de l'intervention du politique sur le religieux, et non l'inverse. Cette séparation n'a pas été immédiatement intégrée, ni même intériorisée ; elle commence à peine, d'ailleurs, à l'être dans la conscience des Japonais comme une valeur fondamentale. Au cours de cette période, emplissant le vide religieux de l'après-guerre et profitant d'une mobilisation forte de la part d'une frange urbaine de la population ayant perdu ses anciennes attaches communautaires, beaucoup de nouvelles religions apparaissent et se développent à un rythme extrêmement rapide, accentuant le pluralisme religieux du pays.

5 La troisième, et dernière période, s'étend des années soixante-dix jusqu'à nos jours. Comme nous l'avons souligné, la neutralisation et la relativisation de la religiosité se sont effectuées dans un contexte spécifique : celui de la mondialisation, de l'individualisation et de la privatisation des pratiques. D'un côté, l'assouplissement et la «banalisation » du culte des ancêtres, que certains sociologues japonais n'hésitent pas - sous l'effet de la sécularisation - à analyser davantage comme un culte des morts ${ }^{2}$, entraînent un nouveau questionnement concernant les notions traditionnelles d'«âme», d'esprit (rei) et de cosmos. Se développe ainsi une recherche radicale de la transcendance du monde de l'existence présente ou d'une dimension sacrale qui s'oppose, pour la première fois, de manière tranchée, à la dimension profane de l'existence. Par ailleurs, liées aux effets conjugués de la mondialisation et de la médiatisation du religieux, la spiritualité japonaise contemporaine se trouve neutralisée et relativisée par cette situation nouvelle.

6 Enfin, si s'offre à chacun, parallèlement au maintien des formes religieuses de la tradition (bouddhisme et shinto), une pluralité de religiosités nouvelles qui ont nom «nouvelles religions » (shinshûkyô), "nouvelles-nouvelles religions » (shin-shinshûkyô), " nouveaux mouvements /nouvelles cultures du monde spirituel »(shin-reisei bunka/undô), il devient à la fois possible de sélectionner dans un vaste registre les éléments susceptibles de convenir à sa propre religiosité, tout en éprouvant de réelles difficultés à maîtriser cette nouvelle dimension du sacré et de la transcendance, à l'intérieur d'une culture religieuse où la démarcation entre les domaines du « sacré » et du « profane » est longtemps restée peu marquée. D'où la tendance, souvent rencontrée, à un recours aux notions traditionnelles de Nippon (le Japon dans son acception de "terre, pays des dieux»), d'empereur, de kami (divinités autochtones), de buddha, de "spiritualité japonaise ", de nature etc., qui semblent, à certains, toujours nécessaires.

7 Ce que nous aimerions présenter dans cet article, c'est l'état des lieux des nouvelles voies spirituelles japonaises, et la mutation religieuse de la société. Cette mutation peut se caractériser, dans le Japon d'aujourd'hui, par deux tendances parallèles : le détachement de la tradition religieuse, d'une part; la restauration indirecte et implicite de la «spiritualité japonaise » sous des formes nouvelles, d'autre part. À la différence de l'Europe chrétienne, le Japon n'a pas connu de système religieux institutionnalisé dominant, mais un ensemble pluriel constitué d'un grand nombre d'écoles de pensée et 
de sectes bouddhistes et shintô qui n'ont joué que rarement -exception faite du shintô d'État de l'ère meijienne - un rôle comparable à celui du christianisme dans les pays de chrétienté.

Quelle est la situation religieuse globale du Japon? La forme principale et traditionnelle de la religiosité populaire japonaise est le syncrétisme shintô-bouddhi-que qui a pour fondement principal le culte des ancêtres et la famille souche traditionnelle (ie). Cette forme religieuse s'apparente à un polythéisme où se mêlent pratiques, croyances et objets rituels du culte shintô et du bouddhisme.

9 Aujourd'hui, les organisations religieuses sont reconnues, en tant que personnalité juridique religieuse (shûkyô hôjin), par la Loi sur les religions de 1951 (modifiée en 1995). Selon l'Annuaire des religions de 1996, publié par l'Agence des affaires culturelles du ministère de l'Éducation, le nombre des groupes religieux indépendants se monte à 183 606 (Shûkyô nenkan, 1996: 1)3. C'est le ministère de l'Éducation, voire les entités territoriales (départements) qui les régularisent et les reconnaissent sans pour autant avoir compétence pour les autoriser. D'après la Loi sur les religions, la dénomination « organisation religieuse » s'applique à tout " groupe » (dantai) qui a pour but principal de transmettre sa doctrine, de pratiquer ses rites, d'éduquer et de guider ses adeptes, et qui possède à cet effet certains lieux de cultes spécifiques, comme des sanctuaires, des monastères, des églises, des couvents et autres institutions similaires ${ }^{4}$.

\section{Les « nouvelles religions » japonaises}

L'histoire des nouvelles religions japonaises est déjà longue de plus d'un siècle et demi, les premières d'entre elles remontant au début du XIX siècle avec, comme référence première, le mouvement d'obédience bouddhique Nyorai-kyô fondé en 1803 à Atsuta (Nagoya). Face au formalisme des religions établies telles que le bouddhisme - parfois nommé « religion des funérailles" par suite de sa gestion quasi exclusive de la mort et des fins dernières - ou le shintô, sous sa forme moderne, le peuple trouve un attrait fort pour des formes religieuses renouvelées, mieux aptes à répondre à ses exigences quotidiennes. Globalement, et dans leur première phase d'expansion, les nouvelles religions s'efforcent de relever un triple défi : celui de la pauvreté et de la maladie, celui du développement extrêmement rapide d'une économie de marché qui tend à marginaliser une frange importante de la population, celui, enfin, de la modernisation et de l'urbanisation de la société japonaise, destructrice des anciens liens communautaires.

Dès lors, le nombre des nouveaux mouvements religieux ne cesse d'augmenter avec des pointes très fortes, mais aussi, parfois, des périodes de stagnation dont il serait intéressant de faire l'historique. Sans entrer dans les détails d'une difficile comptabilisation des «nouvelles religions » - dûe, en partie, à la technique employée (cf. note 3) - et encore moins du nombre de leurs adeptes dont le calcul doit tenir compte de certaines spécificités de la pratique religieuse japonaise (affiliations multiples, tendance à ne pas déclarer défunts ses anciens liens, référence à la famille et non à l'individu comme unité religieuse de base), nous mentionnerons cependant certaines tentatives entreprises pour donner une idée plus précise de l'importance des groupes religieux concernés et du nombre de leurs adeptes. Le Dictionnaire des nouvelles religions japonaises, édité par Matsuno (Matsuno, 1984), mentionne le chiffre de 2000 à 3000 groupes religieux ${ }^{5}$; Shimazono (Shimazono, 1992a : 246) évalue le nombre d'adhérents de ces mouvements à environ 10 à $20 \%$ de la population japonaise, soit 13 à 26 millions de personnes ${ }^{6}$. 

populaires du peuple japonais est le syncrétisme shintô-bouddhique qui a pour assise principale le culte des ancêtres et la «famille souche" traditionnelle (ie). Cette forme religieuse s'apparente à un polythéisme où se mêlent les objets du culte shintô et du bouddhisme. Fondamentalement, les «nouvelles religions» se distinguent autant des croyances populaires que des religions instituées, même si elles empruntent à chacun de ces univers de croyances et de pratiques bon nombre de leurs références.

13

Les religions de l'époque d'Edo ( $\mathrm{du} \mathrm{XVII}^{\mathrm{e}}$ siècle à la fin du $\mathrm{XIX}^{\mathrm{e}}$ siècle), telles le bouddhisme, le shintô, le confucianisme, voire le taoïsme, sont principalement soutenues par les hautes classes de la société, celles des nobles et des guerriers; à l'opposé, les «nouvelles religions » qui naissent à partir du début du XIXe siècle s'appuient sur une base populaire qui voient en elles des religions thérapeutiques ainsi que des religions de salut. Considérées comme les premières religions modernes, leur définition a donné lieu à nombre de discussions dont nous ne pouvons rendre compte dans le cadre de cet article. Nous mentionnerons, cependant, l'étymologie du terme, ses avatars, ainsi que les typologies classiques des sociologues japonais qui ont permis de donner corps à ce concept $^{7}$.

14 Le terme japonais qui désigne ces mouvements - shinshûkyô (littéralement « religions nouvelles ») - est la forme relativement récente qui, à partir des années soixante-dix, remplace le terme plus marqué de shinkô shûkyô («religions nouvellement apparues »), employé, alors, dans deux acceptions différentes : par les journalistes et les médias, pour désigner les mouvements qui, dans $\mathrm{F}$ entre-deux-guerres, étaient dénommés «pseudoreligions » (ryûji shûkyô), à savoir, des religions qui, selon leurs analystes, se donnaient l'apparence de religions mais qui appartenaient, en fait, à un registre moins élevé que celles-ci ; par le Bureau des religions du gouvernement japonais, enfin, qui désignait ainsi les nouvelles organisations religieuses indépendantes du bouddhisme, du shintô et du christianisme ${ }^{8}$.

Les «nouvelles religions » se trouvent à la rencontre de deux courants religieux : celui des religions établies d'une part; celui des croyances populaires d'autre part. Cet emprunt à deux univers religieux existants a donné naissance au terme de «religions syncrétiques" (littéralement: "religions assimilatrices" shûgô shûkyô) qui permet également de désigner certains types religieux anciens comme le shugendô ( $«$ religion des ascètes montagnards ») qui prit, au cours du Moyen Âge, une importance grandissante. Il va sans dire que les "nouvelles religions" revêtiront des formes diverses selon l'importance des éléments intégrés (pratiques magico-religieuses, pensée salvatrice, concepts moraux etc.) $)^{9}$. Parmi les essais de classification les plus souvent formulés, deux typologies sont généralement avancées : la première classe les mouvements par période d'émergence; la deuxième, par inspiration doctrinale. Si aucune des deux n'est fausse, aucune, non plus, n'est satisfaisante. En effet, certains mouvements nés dans une période donnée, n'acquièrent une audience régionale ou nationale que plusieurs dizaines d'années après leur constitution. De même, la nature polymor-phique des nouvelles religions japonaises rend parfois difficile un classement dépendant d'un corps doctrinal facilement identifiable à l'une ou l'autre des grandes traditions religieuses du Japon. Ce qui différencie, en revanche, nettement ces mouvements, c'est qu'ils se présentent comme des religions révélées fondées sur une base volontaire et individuelle, non dépendantes des anciennes appartenances locales (liens du sol dans le cas du shintô, liens familiaux dans celui du bouddhisme). L'allégeance au nouveau groupe religieux n'est 
cependant pas totale. À l'exclusion notoire des mouvements Sôka Gakkai et Tenshô Kôtai jingû-kyô, les adhérents des «nouvelles religions » peuvent conserver leurs affiliations antérieures.

Les principaux traits distinctifs des « nouvelles religions » concernent :

- la réponse apportée à un ensemble de problèmes regroupé sous l'expression générale de " pauvreté, maladie et désordres familiaux » (hin-byô-sô),

- la place de l'écrit dans la diffusion de ces mouvements,

- les enseignements et les pratiques de « la transformation du cœur» (kokoro-naoshi),

- l'importance des liens de type communautaire et des relations humaines,

- le poids mis sur l'existence présente au détriment d'un au-delà peu explicité,

- la conception religieuse du monde qui voit dans l'univers une force nourricière éternelle - « Grande Vie » (daiseimei) - qui donne vie à l'humanité toute entière.

Le premier critère, déjà présent dans les religions historiques japonaises - voir le rôle souvent mésestimé du bouddhisme en tant que religion de guérison -, se trouve au centre de la pensée et de la pratique des religions de salut que sont les «nouvelles religions » : un salut plus proche, plus rapide et « dans le monde ».

Texte et religion ont longtemps connu des rapports distanciés dans les religions populaires japonaises où l'enseignement, gardé secret, reste l'apanage du spécialiste religieux. Mouvements syncrétiques, les «nouvelles religions» ont emprunté aux diverses traditions existantes sur l'archipel : dans le bouddhisme et le shintô -eux-mêmes dépourvus d'une écriture unique, garante d'une orthodoxie imposée -, elles ont puisé un certain nombre de textes auxquels des interprétations nouvelles ont été données dans un langage plus accessible; dans le chamanisme, ensuite, elles ont trouvé un ensemble de traditions locales dont les fondatrices et les fondateurs se sont largement inspirés. Deux modalités nouvelles se sont dégagées de ses divers empunts : le travail sur les textes, au fur et à mesure du processus d'institutionnalisation du mouvement, comme fondement d'une autorité sans cesse interrogée, d'une part; la création massive d'écrits « encyclopédiques » comme moteurs de leur développement et comme voie d'accès à une vérité ou à une technique révélées, d'autre part. Dans le premier cas de figure, il s'agit d'ajuster les textes "fondateurs" aux objectifs annoncés. Souvent de coloration millénariste, les premiers écrits sont révélés par une divinité peu différenciée, et sous la forme d'une injonction dont il serait dangereux de ne pas tenir compte. La fondatrice parle « par la bouche de la divinité » et note ses propos « au fil du pinceau ». Lorsque le groupe religieux accroît le nombre de ses fidèles et que son rayonnement dépasse les frontières villageoises, s'opère, souvent, un travail de réécriture des premiers écrits (réajustement $\mathrm{du}$ message en fonction de l'échéance messianique, mais aussi réorientation du mouvement dont les premiers écrits peuvent être, alors, purement et simplement supprimés). Une attention très vive est portée à la forme légitime du discours écrit, devenu, au fil du temps, « écriture sacrée » et source de valorisation, par la mise en perspective avec une tradition revendiquée. Dans le deuxième cas - celui des « nouvelles religions » contemporaines -, les textes produits, tout en rappelant l'autorité de leur auteur (le ou la fondatrice), dévoilent aussi une conception du monde, un programme religieux, un manuel de mieux vivre voire, plus récemment, une ou plusieurs techniques de réussite individuelle. C'est souvent à la suite de la lecture de ces nombreux écrits, dont une large part est disponible en librairie, que s'effectue l'adhésion dans le groupe religieux ${ }^{10}$. 

marque distinctive de ces mouvements religieux modernes. Elle est constitutive de leurs enseignements et de leurs pratiques. Elle participe d'une discipline des émotions à l'image d'une discipline corporelle. "Kokoro" est un terme difficile à traduire; si son sens littéral est "cœur», il peut également être rendu par "pensée", "sentiment", «émotion », voire " âme ». Helen Hardacre parle même à son égard de « central pillar of the world view of the new religions » (Hardacre, 1986 : 19). En réfléchissant sur soi-même, sur ses comportements passés - en particulier sur la bonté et la politesse envers ses parents, ses ancêtres et ses amis - chacun doit « rectifier » (naosu) son cœur et le rendre meilleur. Ce «travail religieux " s'effectue par soi-même, ou bien grâce à l'aide d'un conseiller religieux, voire de camarades appartenant au même groupe que soi. L'arrièreplan idéologique est la conscience selon laquelle ce sont les manifestations et l'état du « cœur » (kokoro) qui sont cause de malheurs et, qu'en conséquence, la «transformation de son cœur » améliore la vie et les relations humaines, c'est-à-dire, permet d'atteindre au bonheur et d'espérer le salut terrestre.

La création de relations meilleures avec son époux(se), les membres de sa famille et de sa communauté d'origine, ses collègues, ainsi que leur aide réciproque, sont toutes reliées à la notion de salut. L'une des caractéristiques traditionnelles de la religion japonaise est le fait qu'il s'agisse d'une religion des " relations humaines " ${ }^{11}$.

$\mathrm{Au}$ sein des «nouvelles religions", malgré les références à l'au-delà (voire le dernier critère mentionné), l'emphase mise sur l'existence présente est prépondérante. Bien qu'il soit hors de notre propos, dans le cadre de cet article, de commenter le rapport particulier qu'entretient l'au-delà («monde de l'autre rive», anoyo) avec le monde de l'existence présente («monde d'ici-bas », konoyo), dans l'univers religieux populaire, nous aimerions préciser que ce rapport est plus intime, moins distancié qu'il ne l'est dans la culture religieuse de l'Occident. La ligne de démarcation entre les deux univers s'avère peu marquée. Comme l'ont montré les ethnologues des religions - et tout particulièrement Yanagita Kunio dans ses études sur le culte des ancêtres - pour les Japonais, l'au-delà se situe dans un espace proche de l'univers villageois et quotidien ; ces deux espaces communiquant à des moments spécifiques, grâce à la médiation de spécialistes religieux et de divinités spécifiques.

Depuis les années soixante-dix et quatre-vingt sont apparues, au Japon, des formes différentes de "nouvelles religions" que les sociologues japonais ont dénommé, en fonction de leurs caractéristiques propres, des termes peu explicites sur lesquels nous reviendrons, de " nouvelles-nouvelles religions » (shin-shinshûkyô) - abrégées dans la suite de l'article en NNR - pour le premier courant, et de mouvements du « monde spirituel » ( seishin sekai), pour le second, tous deux souvent comparés aux mouvements de type New Age en Occident ${ }^{12}$.

29 En quoi ces types de religiosités récemment apparues se différencient-ils des anciennes «nouvelles religions»? Parallèlement, n'offrent-elles pas nombre de points communs avec la spiritualité japonaise traditionnelle? 


\title{
3. Mutation de la religiosité depuis les années soixante-dix : spécificité idéologique des deux tendances, "religieuses » et " spirituelles »
} sensation de l'entité ou de la force invisible (divinité anonyme) et surnaturelle qui définit un univers holiste, ainsi que par l'unité de l'humanité et de l'univers avec la connaissance et le respect qui en découlent.

\begin{abstract}
qui détermine de façon sociale, historique et culturelle l'attitude envers la spiritualité, le moyen de s'approcher de celle-ci et la relation qu'entretiennent avec elle les individus et la société.
\end{abstract}

\subsection{Les NNR ${ }^{13}$}

Nous allons tenter, à présent, de cerner ces deux nouvelles tendances religieuses et spirituelles qui ont commencé à apparaître dans le Japon des années soixante-dix, en nous efforçant de présenter leurs spécificités idéologiques.

Le sociologue Nishiyama fut le premier, dans la revue Rekishi-kôron, à proposer un terme celui de "nouvelles-nouvelles religions » - et une typologie succincte pour nommer et décrire cet ensemble de croyances et de pratiques qui lui semblaient pouvoir constituer un courant original (Nishiyama, 1979 et 1988). Il allait bientôt distinguer les nouvelles religions, considérées comme des « religions éthiques » (shin no shûkyô), de ces nouvelles manifestations religieuses nommées, de façon antinomique, «religions manipulatrices » (littéralement « religions de la technique - ou de l'art -des esprits » : reijutsu no shûkyô) ou «religions thérapeutiques». Les premières, suite à leurs transformations institutionnelles, auraient mis, selon lui, au centre de leurs dogmes, une sorte de «sacré légitime " proche des religions instituées, alors que dans les secondes, de taille plus réduite, se lirait la réappropriation de pratiques et de croyances délaissées par leurs devancières, sous la forme de la résurgence d'une pensée d'essence millénariste et de cultes qualifiés d'« animistes » ou de « chamani-ques ».

Il va sans dire que nombre de travaux récents critiquent, et cette appellation, et cette typologie peu opératoire, souvent, hélas, en opérant une interprétation réductrice des catégories présentées ${ }^{14}$.

Dans un essai de présentation du renouveau religieux contemporain analysé à travers les «nouvelles-nouvelles religions», Shimazono propose quelques traits spécifiques qui permettraient de distinguer ces mouvements récents des anciennes nouvelles religions (Shimazono, 1992b : 23-50).

Il existerait, tout d'abord, des différences de motivations quant aux raisons qui poussent nombre de jeunes à se joindre à ces mouvements. À la différence des demandes antérieures, essentiellement pragmatiques, il s'agirait, d'une demande visant à combler une solitude, un sentiment de désarroi, d'anxiété et d'ennui dans une société d'abondance où la pauvreté de repères facilement identifiables se fait fortement sentir. 
37 En second lieu, l'originalité des NNR apparaît dans leurs préoccupations, davantage tournées vers le «monde spirituel » - auquel elles se réfèrent en tant que réservoir de sens et de valeurs nouvelles - que vers le monde de l'«existence présente » et des bénéfices immédiats, jusqu'à présent objet de toutes les sollicitudes. Ce détachement du contemporain pour un univers jusqu'alors peu valorisé, ou peu exploité, les différencient, de plus, des «nouvelles religions».

Le troisième point renvoie au concept de «transformation du cœur » qui cesse d'être purement individuel et intériorisé. Les enseignements et les pratiques des NNR insistent sur les techniques psychiques et psychologiques de contrôle du «cœur». Cette technicisation de la «transformation du cœur» cesse de reposer sur une base morale. Shimazono y discerne l'influence d'une pensée positiviste dont l'origine proviendrait du courant de la «nouvelle pensée » (New Thought) américaine.

Quatrièmement, l'augmentation de l'intérêt envers les phénomènes mystiques et les transformations physiques et mentales individuelles sont repérables dans la majorité de ces nouveaux mouvements. Ces expériences mystiques se trouvent valorisées en tant que telles, alors qu'auparavant l'expérience du salut dans la vie quotidienne nécessitait la médiation et la force spirituelle des divinités shintô et bouddhiques. L'expérience mystique est porteuse d'une transformation physique et mentale qui fortifie l'âme, apportant ardeur au travail et confiance en soi.

40 Le cinquième point, la responsabilité individuelle et la conscience de l'éternité de son " esprit-âme ». Le terme " esprit-âme » (reikon), dans son acception traditionnelle, peut se définir ainsi : il s'agit d'une entité spirituelle, généralement invisible, qui s'échappe du corps après la mort et qui peut, soit séjourner dans la montagne ou dans l'au-delà des mers, soit flotter dans les airs ou près de la terre si elle n'est pas suffisamment « consolée » par des rites, des prières et des offrandes appropriées.

41 Si la délivrance des malheurs de ce monde dépend, chez les "nouvelles religions ", des efforts conjoints des individus et des entités surnaturelles, dans les doctrines prônées par les NNR, c'est l'insistance sur la responsabilité individuelle ainsi que la conscience de s'inscrire dans une continuité avec le «monde spirituel » ${ }^{15}$. qui sont mises en avant. Être conscient de sa responsabilité individuelle et posséder la volonté de résoudre les problèmes de son milieu ont une relation de cause à effet sur l'amélioration de sa destinée personnelle. La responsabilité individuelle ne se limite pas au monde de l'«existence présente ». La spécificité de la notion de "responsabilité » à l'intérieur des NNR est intimement liée à l'éternité de l'«âme», parcelle d'un cosmos sacralisé. Cette âme, souvent dénommée tama, indique tout à la fois, l'âme du défunt, l'âme des ancêtres, l'âme divinisée, voire le «principe spirituel» de l'être humain et, dans ce cas, est proche du sens de «cœur» ou d'« esprit » (kokoro). Se lit donc, en arrière plan, une dimension nouvelle, peu présente à l'intérieur des «nouvelles religions ", celle d'une conscience de la réincarnation et d'une pensée karmique qui relient les hommes à leur passé et à leur avenir.

De ceci découle une autre distinction entre les deux courants religieux, à savoir l'intérêt porté par certaines des NNR envers une nouvelle mise en forme du cosmos et des rapports entretenus avec celui-ci. Outre les éléments traditionnels empruntés au bouddhisme de Nichiren, au shintô et au culte des ancêtres, les plus récents des mouvements font volontiers appel à des traditions extérieures - indiennes et tibétaines qui enrichissent leur symbolisme et augmentent le nombre des entités considérées. Elles 
intègrent volontiers dans leur système explicatif du monde, science et religion, notion de transcendance et monisme de l'Univers.

Dernière différence, enfin, l'essor, parmi elles, des millénarismes et des messia-nismes qui avaient pratiquement disparu des "nouvelles religions" à l'intérieur desquelles la «transformation/réparation du monde » (yo-naoshi) avait, peu à peu, laissé la place à une révolution intérieure. Le messianisme des NNR prend les formes les plus diverses, depuis celui de l'attente - voir l'importance des prophéties de Nostradamus dans leurs doctrines (mouvements Kôfoku no Kagaku, Agon-shû, entre autres) - jusqu'à la protestation radicale de type « apocalyptique » du mouvement Aum shinri-kyô ${ }^{16}$.

\subsection{Les « nouveaux mouvements spirituels " (Shinreisei undô) et le "New Age japonais » (Seishin sekai)}

44 Ce deuxième courant spirituel désigne les éléments contemporains d'une recherche spirituelle proche du Nouvel Âge. Ce courant est également appelé «monde spirituel » ( seishin seikai) ${ }^{17}$ parmi les médias et les participants à ces mouvements. Dans cette mouvance du monde spirituel, on inclura la psychothérapie parallèle, la psychologie transpersonnelle, la parapharmacie (aromathérapie, thalassothérapie etc.), les thérapies douces (acupuncture, shiatsu, massage, etc.), l'écologie, la méditation bouddhique (le zen, etc.), le yoga, les arts martiaux (aikidô, taiji-quan), l'« énergie vitale » (qi qong) et la médecine extrême-orientale, la théosophie, l'anthroposophie, le spiritualisme, le chamanisme, le channeling, le Body-Work, etc.

À la différence du premier courant, celui du nouveau monde spirituel est peu structuré. Les affiliations y sont plus lâches et le "nomadisme religieux » plus fréquent. On n'y trouve point de systémisation des dogmes et des pratiques aussi marquée que dans les formes plus anciennes. À ce titre, ces mouvements sont souvent considérés comme des "pseudo-religions ». Outre les livres des fondateurs de ces mouvements, on rencontre, parmi les ouvrages de référence de leurs adeptes, ceux de Krishnamurti, d'éliade et de Jung. Proches du courant du New Age aux États-Unis, les «nouveaux mouvements spirituels » japonais en partagent nombre de caractéristiques communes et constituent une acceptation ou une appropriation du New Age occidental. Ils reposent, cependant, sur un fonds religieux asiatique, et surtout japonais, qui les différencie également des pratiques du New Age. Il est donc nécessaire de séparer - bien que les frontières puissent être assez floues - «monde spirituel» (seishin sekai) et «nouveaux mouvements et cultures du monde spirituel» (shinreisei bunka/undô). Comme nous le verrons, en effet, la "spiritualité japonaise ", au sens large, a des origines multiples : pratiques animistes et shintô ancien, bouddhisme zen, « spiritologie ${ }^{18}$, etc.

Les influences du New Age américain sur la mouvance dite du «monde spirituel» au Japon ont été marquées par plusieurs caractéristiques: «réveil» de la spiritualité, recherche du soi ou de l'authenticité, transformation du soi par des techniques thérapeutiques de type "changer son cœur pour changer le monde ", accentuation de la responsabilité individuelle, importance de la notion de réincarnation - peu prise en compte dans le bouddhisme japonais -, notion de guérison qui se trouve, au contraire, renforcée, idée d'une nécessaire transcendance de la science moderne et de la civilisation occidentale pour établir une nouvelle conscience humaine et une nouvelle civilisation, enfin, représentation d'une cosmologie renouvelée. 
$47 \mathrm{Au}$ cœur des spécificités idéologiques de cette nouvelle culture spirituelle on rencontre les concepts de "Self Spirituality» de Heelas, le «holisme» de Beckford, la «transformation spirituelle individuelle » de Shimazono, l'« enseignement personnel » ( jibunkyô) - proche de ce que Bourdieu nomme l'« autogestion spirituelle » - de Nishiyama, auxquels nous pourrions ajouter, comparable à l'idée de York ${ }^{19}$, la croyance selon laquelle "si on change son cœur, le monde changera ». Il semble qu'à la pensée millénariste des premières «nouvelles religions » dont le slogan tournait autour de la «transformation/ réparation du monde » (yonaoshi) se soit, peu à peu, substituée une pensée davantage centrée sur l'individu, acteur d'une transformation/rénovation personnelle (kokoronaoshi ).

Si les formes organisationnelles des NNR et des mouvements de la nouvelle spiritualité diffèrent - forme en réseau pour les premiers, forme communautaire plus ou moins ouverte pour les seconds -, il est, en revanche, beaucoup plus difficile de dessiner une ligne de partage entre les deux tant les points communs sont nombreux. Nous énoncerons ci-dessous les principaux: égale importance accordée à la sensibilité spirituelle et à l'existence du monde spirituel, supériorité de l'au-delà (ano yo) sur le « monde présent » ( kono yo ${ }^{20}$, recherche d'un autre système de connaissance que le système occidental lié à la rationalité scientifique, engouement pour une cosmologie de type "holiste", individualisation des voies d'accès au sacré, transformation personnelle par la technique de " contrôle du cœur ", prise en charge individuelle et personnelle des problèmes de la vie quotidienne, et, finalement, importance nouvelle accordée à la notion de réincarnation et d'éternité des entités spirituelles (littéralement « esprit-âme ", reikori).

\section{Les deux mutations religieuses majeures des années soixante-dix et quatre-vingt et leurs rapports avec la religiosité japonaise}

49 Avant de présenter ces transformations religieuses contemporaines, il nous semble utile de dresser un tableau succinct de ce que l'on nomme, globalement, la religiosité japonaise traditionnelle qui fut, tout au long des siècles, un inépuisable réservoir de référents religieux.

50 L'un des éléments de base de cette religiosité est l'animisme. À savoir la croyance en des substances immatérielles, «esprits-âmes » des êtres vivants, voire des objets inanimés. Iwata Keiji, un des plus importants ethnologues japonais, a construit une œuvre qui questionne ce concept d'animisme dans lequel il discerne, au-delà d'une énergie ou d'une force spirituelle qui résiderait dans les êtres ou les objets, la racine et le fondement de toutes les religions asiatiques. Cette réinterprétation d'un concept pratiquement disparu du champ des sciences sociales occidentales constitue d'ailleurs l'un des marqueurs d'un nouveau nationalisme culturel qui déborde celui de la spécificité de la culture japonaise, pour s'étendre à l'ensemble de l'aire asiatique (Iwata, 1993) ${ }^{21}$.

51 Le deuxième élément est la pratique d'un syncrétisme religieux qui procède par assimilations successives et dont la forme principale se déploie autour du culte des ancêtres, à travers un ensemble de pratiques et de rites quotidiens visant à mettre en rapport les vivants et les morts. Cette longue familiarité avec un «monde autre » qui apporte protection et assistance est le moyen le plus ordinaire pour ressentir et (re)connaître la présence des « esprits-âmes ». 
52 Troisièmement, c'est la notion de réincarnation, arrière-plan de l'animisme, qui détermine l'attitude envers l'entité invisible et le respect envers les esprits ancestraux. Le quatrième point est le sentiment et la connaissance profonde d'une nécessaire harmonie avec la nature (l'univers). La force nourricière de la nature donne vie à l'humanité qui elle-même s'exprime à travers les multiples figures des relations humaines. Comme le montrent amplement les divers rites calendaires du Japon traditionnel, hommes et plantes appartiennent à un même système. L'opposition nature/ culture apparaît comme relative ; c'est plutôt la première qui englobe la seconde.

Les pratiques chamaniques et méditatives constituent, enfin, les moyens d'accès traditionnels à la spiritualité. Les premières consistent en une communication avec les « esprits » grâce à diverses techniques de possession. Cette communication est souvent médiatisée par des spécialistes religieux différenciés selon les types d'entités avec lesquelles ils entrent en contact (esprit ancestral, divinité protectrice des lieux d'habitation, etc.). Les secondes consistent en l'obtention d'un état spirituel particulier, telle, par exemple, l'illumination (satori) dans la pratique du bouddhisme zen (pratique de la méditation assise), ou bien encore celle d'une ascèse par purification (pratique de l'ascèse des cascades dans les cultes de montagne du shugendô). Parmi ces caractéristiques de la religiosité populaire, c'est l'expression d'un rapport particulier à la nature/univers qui reste, aujourd'hui encore, fondamentale.

Les transformations religieuses récentes tirent une partie de leurs enseignements de ce substrat religieux dont nous venons de tracer les grandes lignes. Les principales ressemblances sont les suivantes :

- Une égale importance accordée à la sensibilité spirituelle et à l'existence d'un monde spirituel ressenti comme proche et sur lequel il est possible d'agir.

57 - Une vision religieuse du monde qui s'appuie sur une cosmologie holiste.

58 - Une technique de « contrôle du cœur » en vue d'une transformation rendue possible par des pratiques de type « manipulationniste ».

59 - Une complémentarité de la notion de réincarnation et d'existence éternelle des « esprits-âmes ».

60 La relative nouveauté de ces mutations religieuses devra être analysée davantage en terme de changements relationnels, de types agrégatifs ou organisationnels inédits, qu'en terme de pratiques et de dogmes. Si le New Age occidental peut sembler marquer une rupture avec le christianisme, dans le cas japonais, cette rupture avec les formes religieuses traditionnelles est moins tranchée. On note souvent des déplacements et des réappropriations d'éléments anciens, oubliés ou peu exploités. Cette revitalisation du religieux contemporain par le recours incessant à des pratiques anciennes offre la même continuité religieuse que celle, de nombreuses fois mentionnée, entre religion populaire et « nouvelles religions ", à la fin du XIX ${ }^{\mathrm{e}}$ siècle.

61 Les mutations religieuses majeures des années soixante-dix et quatre-vingt sont au nombre de quatre :

62 - la première, que nous avons déjà relevée, est l'intérêt croissant porté à la notion d'« audelà » ou, plus exactement, de « vie après la mort » (shigo no sekai) au détriment d'un intérêt religieux essentiellement tourné vers des préoccupations mondaines. Ce nouvel au-delà est à rechercher hors le cercle des grandes civilisations -anciennes ou contemporaines - ou des systèmes politico-économiques présents. Un autre univers de 
connaissance, indépendant de la science occidentale moderne, serait sur le point d'émerger.

- la deuxième mutation est d'ordre personnel. L'importance des relations humaines à

l'intérieur même de l'univers religieux japonais se trouve minorée par rapport à un état spirituel reposant entièrement sur un engagement individuel à travers une multiplication d'expériences. Cet élargissement de la conscience par le recours à un spectre élargi de techniques spécifiques (méditatives, énergétiques, etc.) semblerait en phase avec un individualisme non utilitariste jusqu'à présent en retrait dans la société japonaise, longtemps gouvernée selon des principes communautaires et associatifs.

64 - le troisième changement, qui découle du précédent, est la prise en compte de la responsabilité individuelle comme moyen de résoudre, par soi-même, les problèmes de la vie quotidienne.

Ce qui émerge, aujourd'hui, c'est l'idée selon laquelle la recherche de la spiritualité est une recherche essentiellement individuelle qui marque le point de départ de toute quête d'ordre spirituel, même si cette quête peut aboutir à un engagement collectif. De cette demande personnelle résulte une retenue quant à un engagement fort dans le groupe ou la communauté. Nombre de ces adhésions s'effectuent pour un temps déterminé, à travers des lieux et des relais passagers. À l'opposé, enfin, d'une conception de l'univers fondée sur la représentation d'un cosmos sacré pourvoyeur de bienfaits, les nouveaux mouvements spirituels japonais offrent une alternative à cette vision fixiste qui dénie à l'humanité toute transformation, et par-là, tout progrès. L'image d'une nature éternelle limite, de la sorte, l'idée même de réincarnation et de progrès. On peut discerner, ici, une opposition claire avec la religiosité traditionnelle dont les deux données de base sont le conservatisme et la répétition.

\section{L'arrière-plan socio-culturel et idéologique}

Le milieu socio-culturel à l'intérieur duquel se développe cette nouvelle culture spirituelle n'est pas spécifique à la société japonaise et nombre des phénomènes que nous pouvons y relever sont communs à d'autres aires culturelles. De plus, le processus extrêmement rapide de modernisation et d'urbanisation du Japon, qui entraîna de profondes transformations religieuses et donna naissance à des configurations nouvelles, avait déjà porté un rude coup aux religions de " la famille » (bouddhisme) et du " village » (shintô), dont la fonction allait se redéployer dans la sphère privée.

La stupeur qui saisit le Japon lors de la découverte de l'attentat au gaz sarin perpétré dans le métro de Tôkyô, en mars 1995, par des membres du mouvement Aum shinri-kyô, permit de désigner l'un des maillons faibles du modèle japonais, celui de son système éducatif. Reflet d'une société du diplôme, les étudiants les plus brillants auraient délaissé leurs laboratoires universitaires aux débouchés incertains et à la non-reconnaissance de leurs compétences pour rejoindre une organisation religieuse prête à exploiter leurs jeunes talents. Le groupe Kôfuku no kagaku, sélectionne, quant à lui, les adhérents par un concours d'entrée portant sur la lecture d'ouvrages rédigés par le fondateur, Okawa Ryûhô. Mais, à l'opposé de cette vision, on peut également penser que l'élévation du niveau d'instruction dans le Japon d'aujourd'hui a favorisé cette transformation du religieux au double niveau des groupes considérés et de leurs adhérents. 
68 La défaite des mouvements sociaux et des révoltes étudiantes dans les années soixantedix a également joué un rôle non négligeable dans l'attrait renouvelé d'une classe d'âge qui trouve dans sa participation à des mouvements spirituels nouveaux une compensation à son immobilisme social. C'est, dans un sens, le processus de sécularisation de la société japonaise de l'après-guerre qui a rendu possible, pour une frange jeune de la population, ce nouvel investissement dans des activités de type spirituel où l'emprise sociale, à l'inverse des mouvements politico-religieux des années soixante, est moindre.

L'individualisation des croyances et la privatisation du religieux s'accentuent au cours des deux dernières décennies. La part visible de la religion devient, chez les jeunes, plus ludique. La mise en scène du religieux, grâce aux techniques modernes de communication, accentue la formation de groupes actifs où la religiosité devient spectacle $^{22}$. Parallèlement, les rites religieux qui visaient à la guérison des corps et des âmes sont davantage pris en charge par les individus dont les revendications identitaires deviennent plus fortes à l'intérieur d'une société d'abondance qui, paradoxalement, ne leur offre que peu de repères tangibles.

70 Face aux religions traditionnelles d'une part, dont l'image dans les sondages apparaît de plus en plus négative ou peu différenciée - cas du bouddhisme institutionnel, associé généralement aux seuls rites funéraires -, et aux nouvelles religions d'autre part, dont le nombre d'adhérents semble stagner, voire régresser ${ }^{23}$, s'établissent, en parallèle, des formes de religiosité nouvelles qui accentuent l'écart de plus en plus sensible entre la nostalgie envers une « communauté villageoise » (kyôdôtai) rêvée, et le désir partagé d'un nouvel ordre social qui reste à construire. Nous assistons, semble-t-il, à l'émergence d'un «milieu» nouveau dont les orientations ne sont pas nécessairement d'ordre religieux, d'où le qualificatif, parfois employé à leur encontre, de « culture spirituelle » (reisei bunka ).

71 Le devenir de ces nouveaux courants religieux pose, au sociologue des religions, nombre de questions. Nous mentionnerons, en conclusion, quelques-unes d'entre elles.

72 Cette aventure spirituelle, vécue hors religion organisée, apparait, de prime abord, comme une aventure individuelle. Elle peut valoriser le repli sur soi-même, le détachement des préoccupations sociales et le rejet du politique. Le relativisme des valeurs fait craindre à certains des dérives nationalistes. Les points de rencontre entre la religiosité traditionnelle japonaise et la mouvance spirituelle des années quatre-vingt offre un terrain propice à une pensée ethnocentrique qui se structure autour de l'image du Japon comme terre d'élection d'une "civilisation nouvelle " prête à remplacer la civilisation occidentale et sa rationalité scientifique ${ }^{24}$.

73 Le paysage religieux japonais contemporain apparait ainsi comme extrêmement fluide. Les figures différenciées du "renouveau religieux» (Berthon, 1997) se sont encore diversifiées et doivent être inventoriées avec une rigueur et des moyens plus grands. Si un premier travail d'ensemble a permis de dégager certaines spécificités telle, par exemple, l'importance accordée à l'écrit comme motif d'adhésion, l'analyse de leur structure organisationnelle, entre autres, reste encore à entreprendre.

74 Par ailleurs, une étude récente, pionnière par sa méthodologie, s'est attachée à analyser, de manière globale et sur un espace donné, un ensemble religieux complexe, celui du mont Ikoma (banlieue d'Osaka) et de ses zones environnantes (Shûkyô shakaigaku no kai, 1995). Le maillage religieux de cet espace sur lequel cohabitent religions populaires, 
«nouvelles religions » et communautés ethniques étrangères (coréenne et chinoise), a mis en lumière la prégnance de formes associatives en réseaux, certaines de facture nouvelle, d'autres reprenant à leur compte les anciennes « confréries » $(k o)$ de la religion populaire. Cette tentative, affranchie des contraintes disciplinaires qui avaient, jusqu'à présent, privilégié comme objet d'étude une religion particulière, préfigure, peut-être, la forme que pourraient prendre certaines recherches japonaises dans le domaine des sciences sociales des religions.

\section{BIBLIOGRAPHIE}

BECKFORD James. A., « Holistic Imagery and Ethics in New Religious and Healing Movements », Social Compass, vol. 31, $\mathrm{n}^{\circ}$. 2-3, 1984, pp. 259-272.

BERTHON Jean-Pierre, « Naissance d'une nouvelle religion : La Science du bonheur », France Japon Éco, vol. 49 (nº spécial : « Les Industries culturelles »), 1991.

BERTHON Jean-Pierre, « Religions historiques et "nouvelles religions" japonaises : les figures différenciées du renouveau religieux » in : Catherine CLÉMENTIN-OJHA (textes réunis par), Renouveaux religieux en Asie. Paris, Presses de l'Ecole française d'Extrême-Orient, 1997.

BERTHON Jean-Pierre, «Les mouvements religieux de "réparation du monde" (yonaoshi) » in Actes du colloque franco-japonais «Identités, marges, médiation », Paris, Presses de l'École française d'Extrême-Orient (à paraître).

CHAMPION Françoise, HOURMANT Louis, « Nouveaux mouvements religieux» et sectes » in Françoise CHAMPION et Martine COHEN (éds), Sectes et démocratie, Paris, Seuil, 1999, pp. 68-69.

HAGA Manabu, KISALA Robert J. (éds). « The New Age in Japan », nº spécial de la revue Japanese Journal of Religious Studies, vol. 22, n 3-4, automne 1995.

HARDACRE Helen, Kurozumi-kyô and the New Religions of Japan, Princeton, Princeton University Press, 1986.

HEELAS Paul, The New Age Movement : The Celebration of the Self and The Sacralization of Modernity, Oxford, Blackwell Publishers, 1996.

IKEGAMI Yoshimasa, NAKAMAKI Hirochika (éds). Jôhô jidai wa shûkyô wo kaeru ka (La société de l'information transforme-t-elle la religion ?), Tôkyô, Kôbundô, 1996.

INOUE Nobutaka, « Shin-shin-shûkyô gainen no gakujutsuteki yukôsei ni tsuite'" (Sur la validité scientifique du concept de 'nouvelle-nouvelle-religion') Shûkyô to Shakai, vol. 3, 1997, pp. 3-24.

INOUE Nobutaka et alii. (eds), Shin-shûkyô Jiten (Dictionnaire des nouvelles religions japonaises), Tôkyô, Kôbundô, 1990.

Iто Masayuki, « New Age to seishin-sekai : gainenteki-seiri wo chûshintoshite » (New Age et Monde spirituel : autour d'une classification conceptuelle), « Seishin-sekai no Kôzô » (La structure des mouvements dits du Monde spirituel), papier présenté lors d'un atelier de la réunion annuelle de l'Association japonaise pour l'étude de la Religion et la Société, Tôkyô, 15 juin 1997. 
IWATA Keiji, Sô-moku-chû-gyo no jinruigaku (Anthropologie des plantes, arbres, insectes et poissons). Tôkyô, Tankôsha, 1973.

IWATA Keiji, Animizumu jidai (L'époque de l'animisme). Tôkyô, Hôzôkan, 1993.

KOIKE Kenji, NISHIKAWA Shigenori, MURAKAMI Shigeyoshi (éds), Shûkyô danatsu wo kataru (Récits des oppressions religieuses), Tôkyô, Iwanami, 1978.

комото Mitsugi, « The Place of Ancestors in the New Religions ", Nihon Bunka kenkyûjo kiyô, 62, 1988, pp. 1-31.

LEWIS James R., MELton J. Gordon (éds.). Perspectives on New Age. Albany (N.Y.), State University of New York Press, 1992.

MATSUNo Junkô (éd), Shin-shûkyô jiten (Dictionnaire des nouvelles religions japonaises). Tôkyô, Tokyôdô-shuppan, 1984.

MELTON J. Gordon, New Age Encyclopedia, Detroit (Mich.), Gale Research. 1990.

MORIOKA Kiyomi, « Senzô saishi to Nihon no sezoku-ka » (Rites des ancêtres et sécularisation au Japon), Tôyô gakujutsu kenkyûjo, 25/1, 1986, pp. 43-56.

MURAKAMI Shigeyoshi, Shinshûkyô : Sono kôdô to shisô (Les nouvelles religions japonaises :

comportements et pensées), Tôkyô, Hyôronsha, 1980.

NISHIYAMA Shigeru, « Shin-Shûkyô no genkyô » (La situation actuelle des « nouvelles-nouvelles religions »), Rekishi-kôron, 5-7, 1979, pp. 33-37.

NISHIYAMA Shigeru, « Gendai no shûkyô-undô » (Les mouvements religieux contemporains) in NISHIYAMA Shigeru, OMURA Eishô (eds), Gendai-jin no Shûkyô (La religion de nos contemporains), Tôkyô.Yuhika-ku, 1988.

NISHIYAMA Shigeru, omURA Eishô (eds), « Shinshûkyô no tokuchô to ruikei » (Caractéristiques et typologie des nouvelles religions) in YAMASHITA Kesao (éd.) Nihon shakai-ron no saikentô (Pour un réexamen des théories sociales du Japon). Tôkyô, Miraisha, 1995.

NISHIYAMA Shigeru, OMURA Eishô (eds), « «Shin-shinshûkyô» gainen no gakujutsu-teki yukôsei ni tsuite he no repurai » (Réponse concernant la validité scientifique du concept de «nouvellenouvelle religion »), Shûkyô to Shakai, vol. 3, 1997, pp. 25-29.

SHIMAzono Susumu, «Shin-shûkyô no han.i » (Le domaine des nouvelles nouvelles religions), Shin-Shûkyô Jiten, Tôkyô, Kôbundô, 1990, pp. 5-6.

SHIMAZono Susumu, Gendai kyûsai shûkyô-ron (Théorie sur les religions contemporaines de salut), Tôkyô, Seikyûsha, 1992a.

SHIMAZONo Susumu, Shin-shin shûkyô to shûkyô boom (Les nouvelles-nouvelles religions et le boom religieux), Tôkyô, Iwanami-shoten, 1992 b.

SHimazono Susumu, « New Age and New Spiritual Movements : The Role of Spiritual Intellectuals », SYZYGY : Journal of Alternative Religion and Culture, vol. 2, n 1-2, 1993.

SHIMAzono Susumu, Seishin-sekai no yukue: Gendai sekai to shin-reisei undô, L'avenir du « monde spirituel » : Les nouveaux mouvements spirituels et le monde contemporain) Tôkyô, Tokyôdôshuppan, 1996.

SHIMAZoNo Susumu, «Jidai no chôkô wo yomitoru » (Lire les indices de l'époque), shûkyô to Shakai, vol. 3, 1997, pp. 30-36. 
SHIMIzu Masato (éd.), Shinshûkyô no sekai (Le monde des nouvelles religions), vol. 1, Tôkyô, Daizô shuppan, 1979.

SHIMIzu Masato, Atarashii shûkyô towa nanika (Qu'est-ce qu' une nouvelle religion ?), Tôkyô, Ryôhoku, 1986.

Shûkyô nenkan (Annuaire des religions du Japon), 1996, Bunkachô (éd.) Tôkyô, Gyôsei.

Shûkyô shakaigaku no kai (éd.), Shûkyô nettowâku. Minzoku shûkyô, shin-shûkyô, kakyô, zainichi korian (La religion en réseau. Religions populaires, « nouvelles religions », diaspora chinoise, résidents coréens au Japon), Kyôto, Gyôrô-sha, 1995.

TSUSHIRO Hirofumi, Chinkon gyôhô-ron. Kindai shintô sekai no reikon-ron to shintai-ron (Traité sur les techniques de l'apaisement de l'âme. Théories du corps et des esprits dans le shintô moderne), Tôkyô, Shunju-sha, 1990.

YANAGAWA Keichi, Gendai Nihonjin no shûkyô (La religion contemporaine des Japonais), Kyôto, Hôzôkan, 1991.

YORK Michael, The Emerging Network : A Sociology of the New Age and Neo-Pagan Movements, Londres, Rowman \& Littlefield Publishers, 1995.

\section{NOTES}

1. Sur les persécutions subies par les nouvelles religions pendant l'entre-deux-guerres, voir KOIKE, NISMKAWA et MURAKAMI (éd.), 1978.

2. Cette transformation du culte des ancêtres en milieu urbain constitue l'une des réponses à la question souvent posée : «Quelles sont les figures japonaises de la sécularisation?». Éclairantes sont, à ce propos, les analyses de MORIOKA (1986) - spécialiste du culte des ancêtres - et de комото ( 1988), dont une partie des travaux les plus novateurs concerne la réappropriation et l'élargissement de ce culte au sein des nouveaux mouvements religieux.

3. Le nombre extrêmement élevé d'organisations religieuses répertoriées s'explique par la distinction faite, dans l'Annuaire, entre " groupes religieux unitaires " (tan'itsu shûkyô dantai) et "groupes religieux inclusifs" (hôkatsu shûkyô dantai). les premiers désignent les lieux de culte bouddhistes, shintô, chrétiens etc., souvent inclus dans des organisations plus larges. Les seconds représentent ces groupes de plus grande ampleur, et ont autorité sur eux. Sachant que selon les mêmes statistiques de 1996, il existait sur le territoire japonais 77777 monastères bouddhiques et 85668 sanctuaires shintô, on s'aperçoit que le nombre d'organisations religieuses indépendantes doit être réduit de manière drastique. Ce même annuaire répertorie 1032 organisations religieuses sans que soit distingué leur caractère indépendant ou non, ainsi que 489 organisations religieuses indépendantes. Deux exemples, pris dans l'ensemble bouddhique, illustreront notre propos : la secte zen Sôtô est unique et comprend 14702 monastères; la secte zen Rinzai est subdivisée en 15 écoles qui comprennent au total 5731 monastères. Ces deux sectes représentent ensemble 16 écoles bouddhistes... et 20433 « groupes religieux unitaires ».

4. Deuxième article du chapitre I de la Loi sur la personnalité juridique religieuse (shûkyô hôjin) de 1951, modifiée 1995.

5. Chiffre mentionné dans l'introduction sous forme de question: «le nombre de groupes religieux ne dépasserait-il pas deux à trois mille?». Ce même dictionnaire offre une présentation raisonnée de deux cents «nouvelles religions».

6. Cette "fourchette», hautement problématique, a été établie en se fondant: sur diverses enquêtes nationales concernant la conscience religieuse des Japonais, sur le Dictionnaire des nouvelles religions japonaises de 1990, ainsi que sur une évaluation des membres de la Sôka Gakkai 
(première religion du Japon contemporain par le nombre de ses adeptes) à partir du parti Kômeitô qui en est son émanation politique.

7. Nous nous appuyons, ici, sur les travaux de SHImizu $(1979,1986)$ et de MURAKAMI $(1980)$, ainsi que sur les définitions plus récentes de SHIMAzono (1990) et de NISHIYAMA (1995).

8. Cette différenciation est encore sensible aujourd'hui dans l'Annuaire des religions qui répartit les religions présentes au Japon selon quatre grandes divisions: bouddhisme, shintô, christianisme et «religions autres" avec, cependant, une différence notable : les "nouvelles religions » ne sont pas toutes incluses dans la dernière rubrique, puisqu'il est tenu compte, à présent, du souhait de celles qui demandent à être répertoriées par obédience, voire à être placées dans la rubrique "religions autres", si elles se considèrent comme des religions à tendance universaliste (cas du mouvement Tenri-kyô, depuis 1972).

9. SHIMAZONO, 1992b : pp. 10-14., INOUE et alii, 1990 : pp. 223-227, p. 232.

10. Les titres de certaines "grandes collections" produites par les fondateurs de "nouvelles religions " sont, à cet égard, révélateurs. Nous pensons aux 81 volumes du Reikai monogatari (Histoire du monde des esprits) de Deguchi Onisaburô (mouvement Omoto), à la série Ningen kakumei (Révolution humaine) de Ikeda DAISAKU (mouvement Sôka Gakkai), voire à la somme Seimei no jissô (La réalité de la vie) de Taniguchi MASAHARu (mouvement Seichô no ie) dont le groupe est connu sous l'appellation de « religion éditrice » (shuppan shûkyô). Parmi les écrits plus récents qui jouent à la fois sur les peurs et les angoisses contemporaines, mais aussi sur le besoin de connaissances nouvelles sur les «mondes autres" (monde après la mort, en particulier), à l'aube du troisième millénaire, nous citerons la trilogie des « Lois du salut » que sont Taiyô no hô, Ogon no hô et Eiyû no hô (Lois du Soleil, lois d'Or et Lois de l'Éternité) de Okawa RYûHô (mouvement Kôfuku no kagaku), ainsi que quelques "manuels de réussite» tels Chônôryoku. Himitsu no karikyuramu (Les surcompétences. Un programme d'études secret) de Asahara sHôKô (mouvement Aum Shinri-kyô).

11. Yanagawa fut le chercheur qui insista le plus sur ce particularisme de la religion japonaise. Particularisme qui s'exprime, tout spécialement, dans les manifestations rituelles du religieux, qu'elles soient d'ordre communautaire ou privé (YANAGAWA, 1991, pp. 54-73).

12. Se reporter, à ce propos, à la récente livraison du Japanese Journal of Religious Studies - revue présentant en langue anglaise les recherches japonaises en sciences des religions - intitulée «The New Age in Japan » (HAGA M. et KISALA R. (eds), 1995).

13. Citons, parmi les plus représentatifs, les groupes Shinnyo-en, Agon-shû, Sekai-mahikaribunmei-kyôdan, Sûkyô-mahikari, Aum-shinri-kyô, World-mate, Kôfuku-no-kagaku, Hônohanasanpôgyô.

14. Voir le débat récent dans la revue Shûkyô to shakai (Religion et société), vol. 3, autour de la pertinence scientifique du concept de «nouvelles-nouvelles religions », critiqué par INOUE (1997), et auquel répondent SHIMAzono (1997) et NISHIYAMA (1997). Cette interrogation concernant l'« objet » lui-même, dont la dénomination laisse insatisfait, tout comme les types classificatoires proposés, est un écho lointain, mais très actuel, de préoccupations de nombre de chercheurs français travaillant sur le thème des nouveaux mouvements religieux contemporains [voir la journée d'études du 28 mars 1998 qui s'est tenue à l'École pratique des hautes études, section des sciences religieuses, sur une interrogation semblable ou, tout récemment, les réflexions de Françoise CHAMPION et Louis HOURMANT (1999) sur la validité de la notion de NMR].

15. Ou monde des « âmes » (reikon). Ce terme, difficilement traduisible, participe d'une vision du monde traditionnelle selon laquelle chaque personne est porteuse d'une quantité d' «âmes » entités spirituelles généralement invisibles - qui s'échappent du corps après la mort pour aller séjourner dans des lieux différenciés, plus ou moins lointains, mais qui peuvent également, si elles ne sont pas suffisamment "pacifiées" par des rites, des prières et des offrandes appropriées, rester flottantes dans les airs ou près du sol. 
16. Sur la pensée millénariste au Japon et ses divers modes d'expression, voir BERTHON (à paraître).

17. À partir de la fin des années soixante-dix, la plupart des grandes librairies japonaises ont mis en place un rayon spécial portant cette appellation qu'elles ont contribué, ainsi, à rendre populaire. C'est, aujourd'hui encore, le rayon le plus fourni parmi ceux dévolus à la religion. Outre les ouvrages et magazines religieux, se trouvent placés, sur le même présentoir, tout un ensemble d'objets utilitaires, souvent à caractère ludique, qui accompagnent les enseignements révélés.

18. Traduction du terme japonais reigaku («science des esprits »). Pratique religieuse visant à l'«apaisement de l'âme par un contrôle sur les entités spirituelles»(Chinkon kishin). Cette "science " chamanique, qui s'appuie sur les diverses techniques de manipulation des esprits à l'œuvre dans le shintô, fut développée par Honda Chikaatsu (1822-1889) et son disciple, Nagasawa Katsutate (1858-1940). L'influence de ces techniques chamaniques se retrouve dans nombre de nouvelles religions d'obédience shintô ; entre autres, les mouvements Ômoto, Ananai-kyô et Shindô tenkôkyo. Sur cette « spiritologie » dans le shintô moderne, on lira avec profit l'étude de Tsushiro HIROFUMI (1990).

19. «Un changement majeur et universel (qui est) fondé sur le développement individuel et collectif du potentiel humain » (YORK, $1995: 1-2)$

20. Ceci est une nouveauté sensible par rapport à l'ensemble de la religiosité populaire japonaise qui, selon la pensée des «bénéfices immédiats dans l'existence présente » (genze riyaku), a toujours privilégié, dans ses attentes et ses demandes religieuses, une réponse dans le monde d'ici-bas. On peut d'ailleurs noter la relative pauvreté, au Japon, des doctrines eschatologiques, exception faite du bouddhisme qui, à des fins édificatrices, a présenté à ses fidèles des couches aristocratiques une peinture raffinée de ses multiples paradis. Les premières «nouvelles religions " japonaises qui développèrent une pensée messianique se sont également appuyées sur ce particularisme, tout comme continuent encore de le faire les religions et cultes thérapeutiques.

21. Par-delà le nationalisme religieux présent à l'intérieur des nouvelles religions japonaises ( BERTHON, à paraître), se manifeste également une forme renouvelée de «nippologie » (à savoir, de discours sur le Japon, Nihon-ron) qui interroge les manifestations contemporaines du croire. La spécificité d'une religiosité asiatique devient le vecteur d'une tradition culturelle commune dont l'animisme offrirait l'un des fondements. Cette religion «primitive/première » de l'Asie, loin de représenter la première étape d'un assemblage religieux de type évolutif, constituerait, en fait, l'essence même d'un religieux contemporain « redécouvert ». Voir, à ce sujet, un autre ouvrage d' IWATA : Anthropologie des plantes, arbres, insectes et poissons (IWATA, 1973).

22. La Fête des étoiles (Hoshi matsuri) du mouvement Agon-shû rassemble, chaque année, près de 500000 personnes dans les montagnes de l'est, à Kyôto, pour un spectacle, retransmis par satellite, où se mêlent bouddhisme ésotérique et culte des montagnes. Depuis quelques années, Okawa Ryûhô, le fondateur du mouvement Kôfuku no kagaku, loue le stade couvert de base ball du quartier de Suidôbashi, à Tôkyô, pour y célébrer son anniversaire, devant 30 à 35000 fidèles ( BERTHON, 1991). Concernant l'impact des techniques de communication sur les religions japonaises, voir IKEGAMI et NAKAMAKI (1996).

23. La comparaison des chiffres présentés dans l'Annuaire des religions depuis les années cinquante jusque dans les années quatre vingt-dix, laisse apparaître une stagnation, voire un déclin, des plus importantes nouvelles religions japonaises: Sôka gakkai, Risshô kôsekai, Reiyûkai, Seichô no le, PL. Kyôdan, Tenri-kyô, entre autres.

24. Dans un article récent consacré à ce phénomène, Shimazono forge le terme d'« intellectuels spirituels " - par opposition aux intellectuels religieux, bouddhistes ou chrétiens - et présente quelques-uns de leurs écrits (shimAzono 1993). Peut se lire également, dans cette nouvelle 
littérature, une forme renouvelée des nippologies des années soixante-dix dont le slogan était celui de l'unicité et de la spécificité de la culture japonaise. Certaines «nouvelles-nouvelles religions ", et le mouvement Kôfuku no kagaku tout particulièrement, insistent d'ailleurs sur l'importance d'un message messianique et universalistequi accorde au Japon une place centrale pour ne pas dire la première - dans le nouvel ordre mondial.

\section{RÉSUMÉS}

À partir de la deuxième moitié des années soixante-dix, l'émergence de formes nouvelles de croyances et de pratiques religieuses dans la société japonaise, a relativisé la distinction communément admise entre "religions historiques", "nouvelles religions " et "croyances populaires ». La présente étude vise à offrir un état des lieux de ces religiosités, et à dresser le tableau en «fondu-enchaîné » d'un univers religieux à l'intérieur duquel le syncrétisme shintobouddhique s'est trouvé enrichi de références qui s'appuient sur des traditions jusqu'ici peu explicitées, voire étrangères au fonds religieux national.

Ces mouvements spirituels marquants ne doivent cependant pas faire oublier le constant processus de réinterprétation et de revitalisation du religieux traditionnel qui continue d'alimenter la mutation religieuse actuelle, et qui rend le cas japonais digne d'intérêt. C'est cette double orientation que les auteurs ont essayé de formaliser.

From the second half of the seventies, new manifestations of religious beliefs and practices in contemporary Japanese society have relativized the commonly used distinction between "historical religions", "new religions" and "popular beliefs". The present article aims at offering a picture of these religiosities and at drawing up an inventory of a religious universe in which shinto-buddhist syncretism has been enriched by new elements, until recently not clarified or even foreign to the national religious background.

However, these new spirituals movements must not refrain us to remember the constant process of reinterpretation and reinvention of traditional religiosity that continues to feed the actual religious changes, and that makes the Japanese case worthy to study. This is this twofold orientation that the authors of the article have tried to formalize.

A partir de la segunda mitad de los años 70, la aparición de nuevas formas de creencias y de prácticas religiosas en la sociedad japonesa obligé a relativizar la distinción común que se admite entre 'religiones históricas', 'nuevas religiones' y 'creencias populares'. El presente estudio intenta ofrecer un panorama de estas religio-sidades y construir un cuadro de tipo 'fundidoenlace' de un universo religioso en el seno del cual el sincretismo shinto-bûdico se vio enriquecido por referencias surgidas de tradiciones que estaban hasta la fecha poco explicitadas o eran extranjeras al fondo religioso nacional.

Estos movimientos espirituales significativos no deben por lo tanto hacer olvidar el proceso constante de reinterpretación y de revitalizaciôn del religioso tradicional, que sigue alimentando la mutación religioso actual, y que hace del caso japonés un caso de mucho interés. Los autores trataron de formalizarlo en esta doble orientación. 


\section{AUTEURS}

JEAN-PIERRE BERTHON

CNRS-Paris

NAOKI KASHIO

Université Keiô (Tôkyô) 